\title{
Variação da composição e abundância das espécies da Classe Appendicularia e seu uso como potenciais bioindicadoras de regiōes e massas de água superficiais na área da Bacia de Campos, Rio de Janeiro, Brasil
}

\author{
Pedro F. de Carvalho \& Sérgio L. C. Bonecker
}

Laboratório Integrado de Zooplâncton e Ictioplâncton, Departamento de Zoologia Instituto de Biologia Universidade Federal do Rio de Janeiro, 21941 -590 Rio de Janeiro, RJ, Brasil. (pedropfcilha@yahoo.com.br)

Recebido 29 fevereiro 2016.

Aceito 29 agosto 2016.

DOI: $10.1590 / 1678-4766 \mathrm{e} 2016022$

\begin{abstract}
Variation of species abundance of Class Appendicularia and their use as potential bioindicators of the regions and surface water mass in the area of the Campos Basin, Rio de Janeiro, Brazil. Studies about the relationship of the species of appendicularians with spatial gradients and water masses are rare, especially in the South Atlantic. The present study aims to fill the knowledge gap about the abundance of the community of appendicularians and on their bioindicator function in different regions (continental shelf and slope), with influence of different water masses in the South Atlantic. Sampling was conducted in the rainy season (February-April 2009) and the dry season (August-September 2009) in the subsurface layer. The water masses found on the continental shelf of the study area were the Tropical Water (AT), which was prevalent during the rainy season, and the Coastal Water (AC), which was prevalent in the dry season. In both sampling periods has not registered the presence of SACW on the surface over continental shelf. The appendicularians were more abundant in the region of the continental shelf influenced by the CW. A total of 18 species of appendicularian was registered. Oikopleura longicauda Vogt, 1854 and O. fusiformis Fol, 1872 were the most abundant appendicularian. Oikopleura dioica Fol, 1872 was found exclusively in the continental shelf on the CW. In despite of the SACW water had not registered on the surface, the presence of, high values of density of appendicularians in front of Cabo Frio region suggest the influence of an upwelling event in a anterior period in the region. In relation to the appendicularians abundance, high values were also observed in front of the Paraíba do Sul river with the CW influence. This fact suggests a high nutritional contribution of this river in the coastal marine area adjacent. The spatial variation and different water masses influenced the abundance and the composition of appendicularians. Among the species identified, only O. longicauda was considered as a bioindicator of the CW.
\end{abstract}

KEYWORDS. Zooplankton, Oikopleura, Fritillaria, Southwestern Atlantic.

RESUMO. Estudos sobre a variação espacial das espécies de apendiculárias e sua relação com as massas de água são raros, principalmente no Atlântico Sul. $\mathrm{O}$ atual estudo pretende preencher a lacuna de conhecimento sobre a abundância, composição específica e a função de bioindicadora das apendiculárias, em diferentes regiões (plataforma continental e talude) e massas de água, no Atlântico Sul Ocidental. As coletas foram realizadas no período chuvoso (de fevereiro a abril de 2009) e no período seco (de agosto a setembro de 2009) na camada subsuperficial. As massas de água encontradas na superfície na plataforma continental da área de estudo foram a Água Tropical (AT), que predominou no período chuvoso, e a Água Costeira (AC), que predominou no período seco. Em ambos os períodos de coleta não foram registradas a presença da Água Central do Atlântico Sul (ACAS) na superfície na plataforma continental. As apendiculárias foram mais abundantes na região de plataforma continental influenciada pela AC. Foi identificado um total de 12 espécies da Classe Appendicularia. Oikopleura longicauda Vogt, 1854 e O. fusiformis Fol, 1872 foram as espécies mais abundantes. Oikopleura dioica Fol, 1872 foi encontrada exclusivamente na plataforma continental na AC. Apesar não ser observado a presença de ACAS na superfície, altos valores da densidade de apendiculária em frente a Cabo Frio sugerem a influência de uma pós-ressurgência no local. Também foram observados valores elevados na abundância das apendiculárias em frente ao rio Paraíba do Sul, com influência da AC. Este fato sugere a alta capacidade de incremento de nutrientes desse aporte fluvial. A variação espacial e as diferentes massas de água influenciaram a abundância e a composição das apendiculárias. Dentre as espécies identificadas, somente $O$. longicauda foi considerada como bioindicadora da AC.

PALAVRAS-CHAVE. Zooplâncton, Oikopleura, Fritillaria, Atlântico Sul Ocidental.

As apendiculárias estão entre os organismos mais comuns do zooplâncton e são amplamente distribuídas em todos os oceanos do mundo (CAPITANIO et al., 2005), possuem aproximadamente 64 espécies, nas quais 43 já foram registradas para o Atlântico Sul (ESNAL, 1999; BOLTOVSKOY et al., 2003). A maioria dos estudos com apendiculárias são sobre a distribuição, abundância e diversidade nas regiões costeira, de plataforma e oceânicas do Brasil e do mundo (p. ex. Aravena \& Palma, 2002; Hidaka, 2008; Bonecker \& Carvalho, 2006; Carvalho \& Bonecker, 2010; VegaPÉREZ et al., 2011).

Existem poucas informações sobre a utilização das apendiculárias como indicadores hidrológicos (EsNAL, 1999). Alguns autores caracterizam as espécies de apendiculária 
como boas bioindicadoras de massas de água (ForNeris, 1965; HsiaO et al., 2011). Algumas espécies mostraram uma preferência por águas continentais com mistura de águas oceânicas no Atlântico Sul Ocidental (BJöRNBERG \& ForNERIS, 1956; CAPITANIO \& ESNAL, 1997) apesar disso, pouco se conhece sobre a variação espacial (plataforma e talude), sobre as preferências hidrológicas e a função de bioindicadoras das espécies de apendiculárias.

A identificação de espécies características ou indicadoras é uma atividade tradicional em ecologia e em biogeografia (DUFRÊNE \& LEGENDRE, 1997). Espécies zooplanctônicas, que incluem as apendiculárias, são boas indicadoras de condições ambientais e de massa de águas (EsPINO, 2000).

A Bacia de Campos está localizada ao sul do estado do Espírito Santo $\left(40^{\circ} 20^{\prime} 3,872^{\prime \prime W}, 20^{\circ} 26^{\prime} 37,232^{\prime \prime} \mathrm{S}\right.$ e $\left.37^{\circ} 12^{\prime} 53,768^{\prime \prime} \mathrm{W}, 21^{\circ} 59^{\prime} 33,737^{\prime \prime} \mathrm{S}\right)$ e ao norte do estado do Rio de Janeiro $\left(42^{\circ} 0\right.$ ' $42,944^{\prime \prime} \mathrm{W}, 23^{\circ} 0^{\prime} 48,576$ ”'S e $40^{\circ} 32^{\prime} 29,119^{\prime \prime} \mathrm{W} 25^{\circ} 21^{\prime} 43,277^{\prime}$ 'S), ocupando uma área de mais de $100.000 \mathrm{~km}^{2}$ (VIANA et al., 1998). A plataforma continental mede cerca de $100 \mathrm{~km}$ e a quebra da plataforma entre a isobata de 80 e $130 \mathrm{~m}$ nas porções norte e sul, respectivamente. $\mathrm{O}$ talude tem uma extensão de até $40 \mathrm{~km}$ de largura e possui um declive médio de $2,5^{\circ}$ (Dias et al., 2015). A região da Bacia de Campos está localizada dentro de duas províncias biogeográficas, uma com água oligotrófica com influência costeira e outra pelo fenômeno de resurgência localizada na área de Cabo Frio (GonZalez-RodrigueZ et al., 1992). Esta região é influenciada diretamente pela Corrente do Brasil (CB), que está associada ao Giro Subtropical do Atlântico Sul que se origina ao sul de $10^{\circ} \mathrm{S}$, na região onde a Corrente Sul Equatorial (CSE) se bifurca formando também a Corrente do Norte do Brasil (CNB) (Silverra et al., 1994). As massas de água que influenciam a camada superficial da plataforma continental da Bacia de Campos são Água Tropical (AT) carregada pela Corrente do Brasil, Água Costeira (AC) que é a mistura dá água de plataforma com a água de origem continental e a Água Central do Atlântico Sul (ACAS) que é o resultado da ressurgência de Cabo Frio (Silveira et al., 2000).

O objetivo deste estudo foi avaliar as diferenças da densidade e composição das espécies da Classe Appendicularia entre as regiões de plataforma e talude, levando em consideração as diferentes massas de água que influenciam a camada superficial da região da plataforma continental na Bacia de Campos, buscando bioindicadores de áreas ou de massas de água, em dois períodos de estudo.

\section{MATERIAL E MÉTODOS}

As amostras utilizadas fazem parte do Projeto Habitats - Heterogeneidade Ambiental da Bacia de Campos coordenado pelo CENPES/PETROBRAS, que visou estudar as diferentes comunidades zooplanctônicas na região da Bacia de Campos, ao longo da coluna de água. O presente estudo analisou apenas o primeiro estrato, um metro de profundidade visando analisar as variações espaciais superficiais.
As coletas foram realizadas no período chuvoso (de fevereiro a abril de 2009) e no período seco (de agosto a setembro de 2009) em 48 estações divididas em duas regiões: plataforma (isóbatas $1-25 \mathrm{~m}, 2-50 \mathrm{~m}, 3-75 \mathrm{~m}$ e $5-150$ $\mathrm{m}$ ) e talude (isóbatas $6-400 \mathrm{~m}, 8-1000 \mathrm{~m}, 10-1900 \mathrm{~m}$ e $12-3000 \mathrm{~m}$ ) distribuídas em seis transectos nomeados de sul pra norte (A, C, D, F, H e I) (Fig. 1).

Para determinar as massas de água na superfície das estações na coluna de água foi perfilada com o CTD (Seabird $\left.{ }^{\circledR}\right)$ para obtenção de medições in situ de temperatura, salinidade e condutividade. As massas de água superficiais foram determinadas pelos parâmetros salinidade e temperatura. A massa de água com salinidade acima de 36,2 e temperaturas acima de $20^{\circ} \mathrm{C}$ foi considerada AT e salinidade abaixo de 36,2 foi considerada AC, baseado no estudo de MÉMERY et al. (2000). Temperaturas abaixo dos $20^{\circ} \mathrm{C}$ foi considerada ACAS, baseado no estudo de BoNECKER et al., 2014)

Para obtenção das apendiculárias foram realizados arrastos horizontais a um metro de profundidade, no período noturno com rede MultiNet ${ }^{\circledR}$ (Hydro-Bios, $200 \mu \mathrm{m}$, com $50 \mathrm{x}$ $50 \mathrm{~cm}$ de abertura de boca). Para o cálculo da densidade, um fluxômetro eletrônico acoplado à boca da rede foi utilizado para calcular o volume de água filtrada. As amostras foram preservadas em formol a $4 \%$ tamponado com bórax.

No laboratório os exemplares foram fracionados com auxílio do Folsom Plankton Sample Splitter ${ }^{\circledR}$ (Hydro-Bios) e triados até um mínimo de 100 indivíduos. A identificação foi realizada com o auxílio da bibliografia (EsNAL, 1999; Aravena \& Palma, 2002; Bonecker \& Carvalho, 2006). As espécies encontradas estão tombadas na coleção zoológica do Laboratório Integrado de Zooplâncton e Ictioplâncton (DZ/UFRJ).

Para testar se a densidade das apendiculárias apresentou diferenças $(p<0,05)$ em relação às regiões de amostragens (plataforma e talude) e às massas de água sobre a plataforma continental (AC e AT), foi aplicado o teste de Kruskal-Wallis. Para testar o efeito das mesmas variáveis sobre a composição das espécies, foi efetuada a análise de similaridade ANOSIM (comunidades diferentes quando o Nível de significância $<0,5 \%$ ). Os testes citados acima foram conduzidos separadamente para o período chuvoso e seco.

Para verificar a preferência das espécies pelas diferentes regiões (plataforma e talude) e pelas diferentes massas de água da plataforma continental (AT e AC) foi aplicado o teste Indicator Species Analysis (ISA) (DUFRÊNE \& LEGENDRE, 1997). Foram consideradas indicadoras as espécies que apresentaram os valores de $p=<0,05$ e os valores de IndVal acima de $70 \%$. A análise foi realizada no programa PCORD 5.

\section{RESULTADOS}

As apendiculárias foram mais abundantes na região de plataforma nos dois períodos de coleta. A maior densidade média foi observada durante o período seco $\left(241,86\right.$ ind. $\mathrm{m}^{-3}$ - DP 808,47 ind. m $^{-3}$ ) (Fig. 2). A variação das densidades das apendiculárias entre as regiões de plataforma e talude nos dois 
períodos apresentaram diferenças significativas $(\mathrm{P}=0,00)$.

Foram identificadas 12 espécies de apendiculária, sendo oito pertencentes a Oikopleuridae e quatro a Fritillaridae (Tab. I). Oikopleura dioica e Fritillaria sargassi foram exclusivas da área de plataforma e $O$. albicans foi exclusiva do talude. A espécie mais abundante foi O. longicauda, representando $96,78 \%$ na região de plataforma durante o período seco. Oikopleura rufescens foi a segunda mais abundante, e seu pico de abundância foi observado no talude durante o período chuvoso com $8,41 \%$.

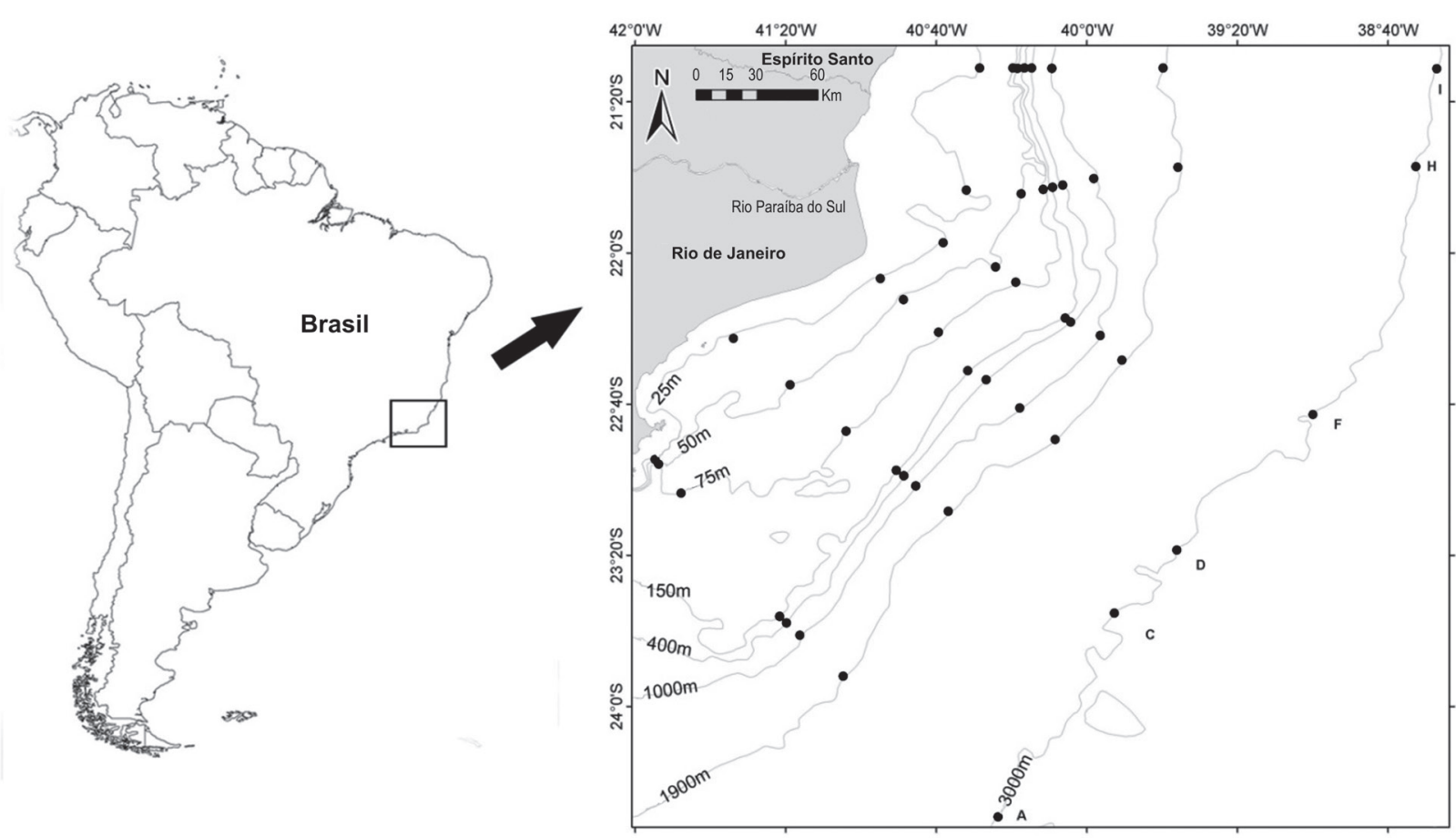

Fig. 1. Localização da Bacia de Campos, RJ, Brasil com as estações de amostragem pesquisadas neste estudo, suas isóbatas $(25 \mathrm{~m}, 50 \mathrm{~m}, 75 \mathrm{~m}, 150 \mathrm{~m}$, $400 \mathrm{~m}, 1000 \mathrm{~m}, 1900 \mathrm{~m}$ e $3000 \mathrm{~m}$ ) e seus transectos (A, C, D, F, H e I).

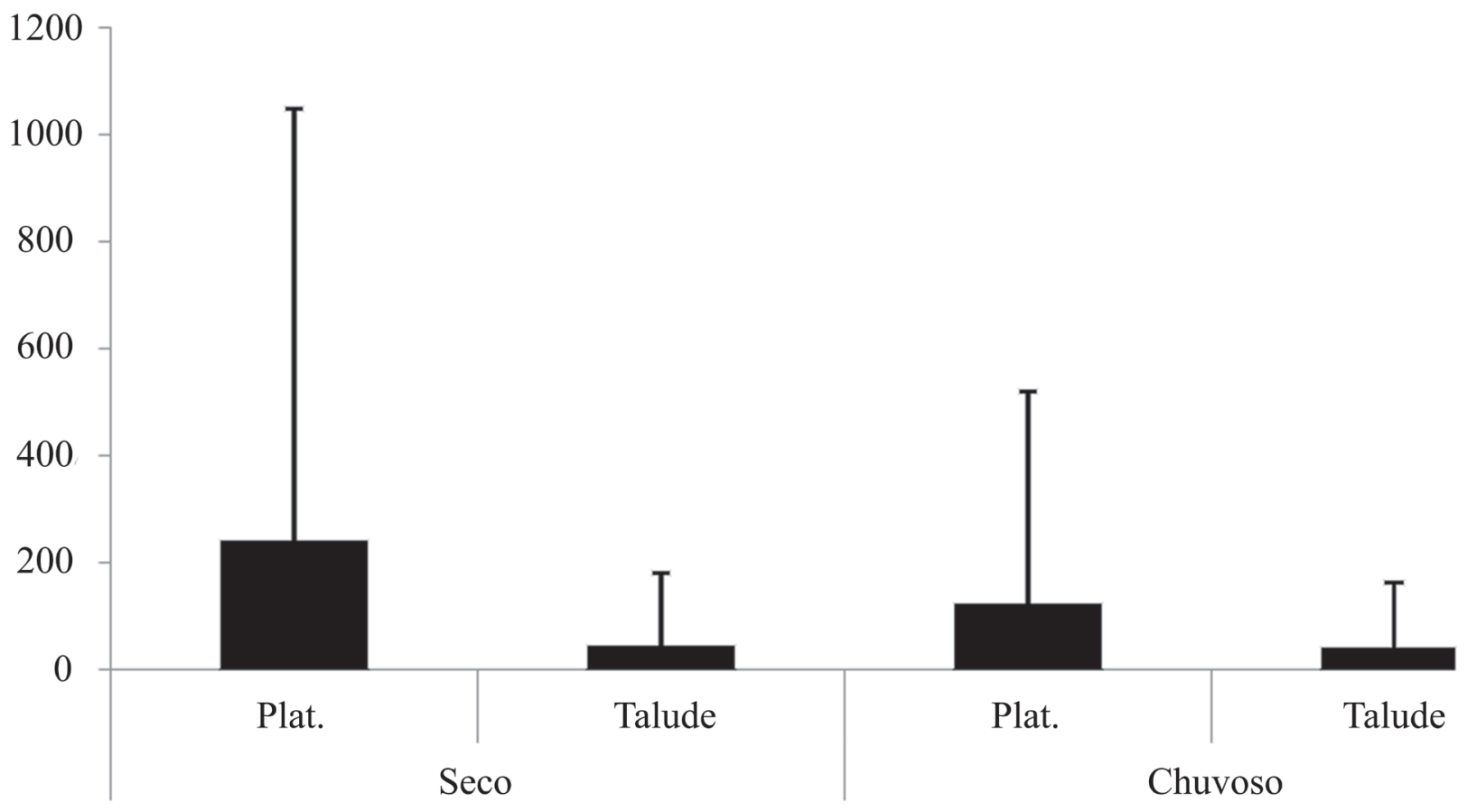

Fig. 2. Densidade média (barra preta) e desvio (traço vertical) padrão das apendiculárias (ind.m-3) nas duas áreas (plataforma e talude) durante os dois períodos de coleta (seco e chuvoso), Bacia de Campos, RJ, Brasil. 
Tab. I. Densidade média e desvio (DP) (ind. $\mathrm{m}^{-3}$ ) e abundância relativa das espécies de apendiculária coletadas na plataforma e no talude durante os dois períodos de coleta na área de estudo, Bacia de Campos, RJ, Brasil.

\begin{tabular}{|c|c|c|c|c|c|c|c|c|c|c|c|c|}
\hline & \multicolumn{6}{|c|}{ Período Chuvoso } & \multicolumn{6}{|c|}{ Período Seco } \\
\hline & \multicolumn{3}{|c|}{ Plataforma } & \multicolumn{3}{|c|}{ Talude } & \multicolumn{3}{|c|}{ Plataforma } & \multicolumn{3}{|c|}{ Talude } \\
\hline & Média & DP & $\%$ & Média & DP & $\%$ & Média & DP & $\%$ & Média & DP & $\%$ \\
\hline OIKOPLEURIDAE & 57,52 & 116,04 & 94,70 & 17,95 & 16,62 & 90,54 & 117,04 & 320,01 & 96,78 & 21,61 & 40,32 & 87,31 \\
\hline Oikopleura longicauda Vogt, 1854 & 0,85 & 1,30 & 1,40 & 1,67 & 2,21 & 8,41 & 0,37 & 1,19 & 0,30 & 0,18 & 0,31 & 0,74 \\
\hline Oikopleura rufescens Fol, 1872 & 0,80 & 1,61 & 1,32 & 0,09 & 0,23 & 0,44 & 0,95 & 2,12 & 0,79 & 0,88 & 2,10 & 3,55 \\
\hline Oikopleura fusiformis Fol, 1872 & 0,00 & 0,02 & 0,01 & 0,00 & 0,00 & 0,00 & 1,31 & 3,87 & 1,08 & 1,27 & 3,71 & 5,12 \\
\hline Oikopleura cophocerca (Gegenbaur, 1855) & 0,00 & 0,00 & 0,00 & 0,00 & 0,00 & 0,00 & 0,00 & 0,00 & 0,00 & 0,02 & 0,04 & 0,06 \\
\hline Oikopleura albicans (Leuckart, 1854) & 0,00 & 0,00 & 0,00 & 0,00 & 0,00 & 0,00 & 0,10 & 0,47 & 0,08 & 0,09 & 0,40 & 0,38 \\
\hline Oikopleura cornutogastra Aida, 1907 & 0,00 & 0,00 & 0,00 & 0,00 & 0,00 & 0,00 & 0,19 & 0,82 & 0,16 & 0,00 & 0,00 & 0,00 \\
\hline Oikopleura dioica Fol, 1872 & 0,49 & 1,30 & 0,81 & 0,12 & 0,31 & 0,62 & 0,14 & 0,31 & 0,12 & 0,23 & 0,71 & 0,94 \\
\hline FRITILLARIDAE & 0,96 & 4,64 & 1,58 & 0,00 & 0,00 & 0,00 & 0,79 & 2,10 & 0,66 & 0,26 & 0,67 & 1,07 \\
\hline Fritillaria formica Fol, 1872 & 0,07 & 0,34 & 0,11 & 0,00 & 0,00 & 0,00 & 0,02 & 0,08 & 0,02 & 0,01 & 0,03 & 0,03 \\
\hline
\end{tabular}

A maioria das espécies apresentou maior densidade média em águas da região de plataforma, como $O$. longicauda, $O$. fusiformis, O. intermedia, O. dioica, F. pellucida, $F$. haplostoma e $F$. sargassi. Para outras espécies os maiores valores de densidade média foram encontrados no talude, como $O$. cophocerca e $O$. albicans. Os maiores valores de densidade média de $O$. rufescens e $F$. formica variaram entre as regiões dependendo do período do ano, a primeira o maior valor ocorreu no talude durante o período chuvoso e na plataforma durante o período seco, e a segunda apresentou o padrão inverso (Tab. I). As variações na composição da comunidade das apendiculárias foram significativamente diferentes entre as regiões de plataforma e talude (nível de significância $=0,2 \%$ ).

Foram observadas duas massas de água na superfície da região da plataforma continental na área de estudo: AT (salinidade - máxima 37,3 e mínima 36,3; temperatura máxima $28,3^{\circ} \mathrm{C}$ e mínima $21,3^{\circ} \mathrm{C}$ ) e outra que predominou em quase todas as estações da plataforma, AC (salinidade
- máxima 36,2 e mínima 35,4; temperatura - máxima $26,1^{\circ} \mathrm{C}$ e mínima $19,6^{\circ} \mathrm{C}$ ). Não foi observada a presença da massa de água ACAS na superfície da plataforma continental em nenhum dos dois períodos de estudo, nem mesmo na região localizada em frente a Cabo Frio. As águas com temperaturas inferiores a $18^{\circ} \mathrm{C}$, típicas da ACAS, ficaram restritas a uma lâmina delgada próxima ao fundo, sendo que as temperaturas próximas à superfície ficaram entre $28,3^{\circ} \mathrm{C} \mathrm{e} 19,6^{\circ} \mathrm{C}$ (Fig. 3).

Na região de plataforma durante o período chuvoso as apendiculárias foram mais abundantes na $\mathrm{AC}$ na estação A3, com 733,87 ind. $\mathrm{m}^{-3}$ (Fig. 4). A variação das densidades das apendiculárias entre as duas massas de água (AC e AT) presentes na plataforma apresentaram diferenças significativas no período chuvoso $(\mathrm{P}=0,00)$. Durante o período seco as apendiculárias foram mais abundantes na AC na estação $\mathrm{H} 5$, com 1574,21 ind. $\mathrm{m}^{-3}$ (Fig. 4). A variação das densidades das apendiculárias entre as massas de água AC e AT presentes na plataforma não apresentaram diferenças significativas no período seco $(\mathrm{P}=0,97)$.
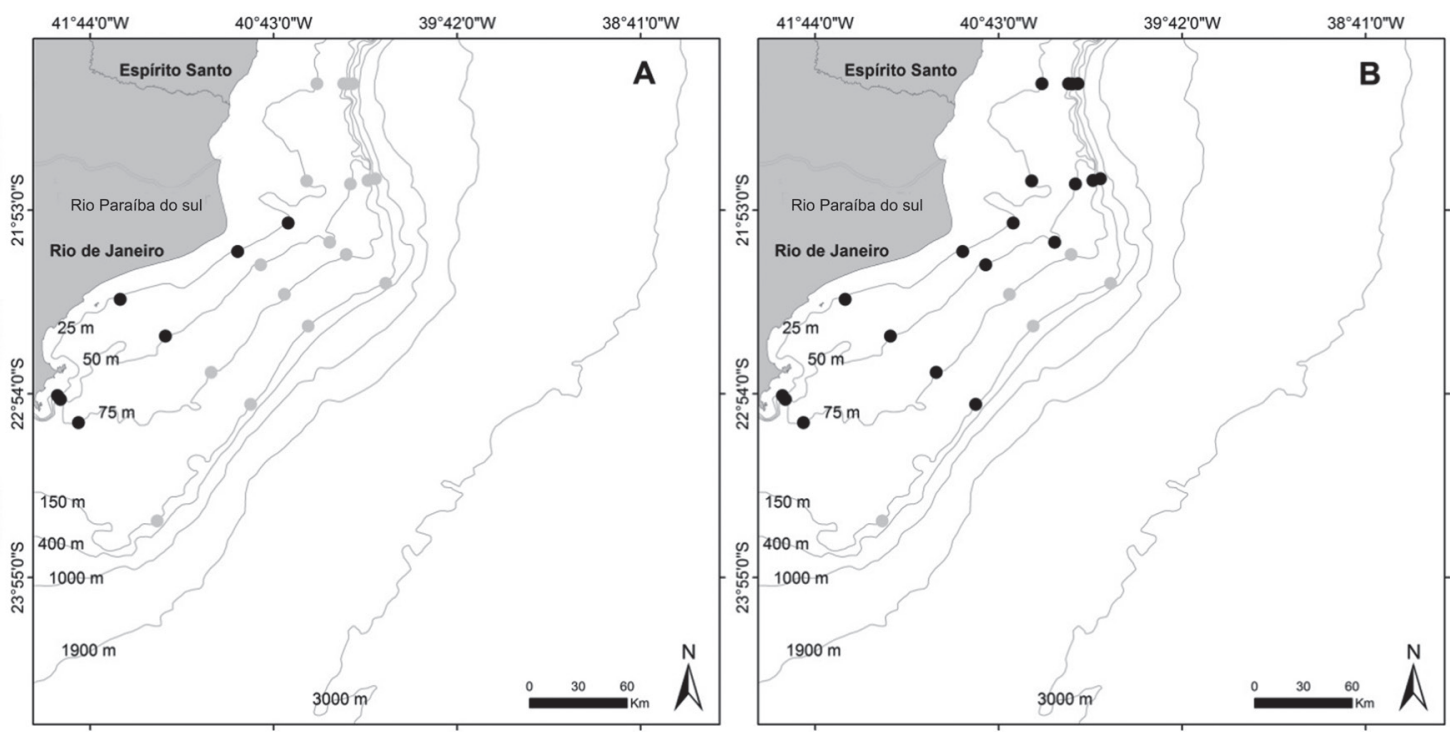

Fig. 3. Distribuição das estações na plataforma continental da Bacia de Campos, RJ, Brasil (A, período chuvoso e B, período seco) indicando as estações sob influência da AC (Águas Costeiras) = bolas pretas e AT (Águas Tropicais) = bolas cinzas. 


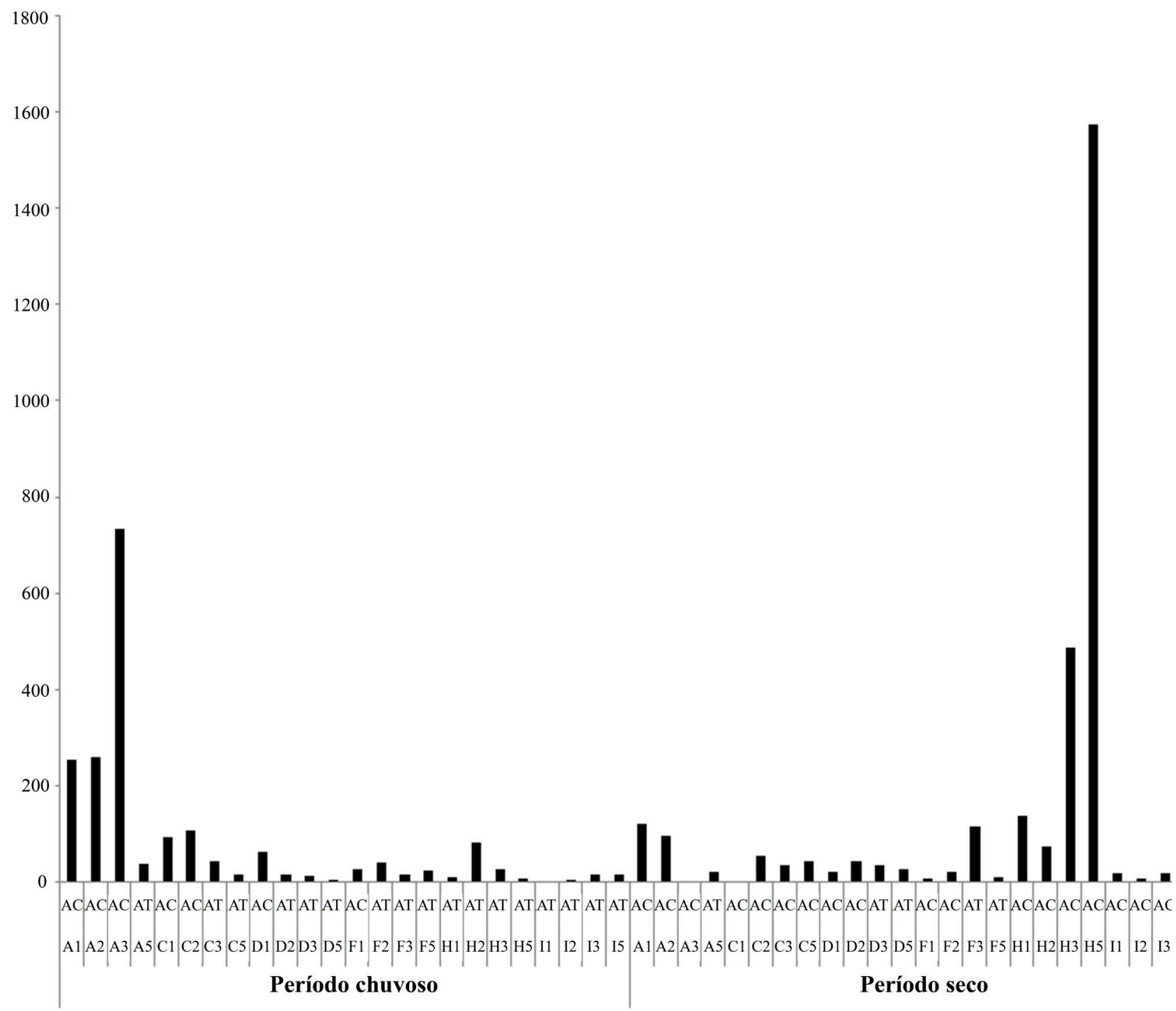

Fig. 4. Densidade das apendiculárias (barra preta - ind.m-3) entre as estações de coleta (transectos A, C, D, F, H e I e isóbatas 1, 2, 3, 5) e as massas de água $($ Água costeira = AC e Água Tropical = AT) que estiveram presente na plataforma durante os períodos chuvoso e seco, Bacia de Campos, RJ, Brasil.

Tab. II. Densidade Média (ind.m-3), desvio padrão (ind.m-3) e abundância relativa das espécies de apendiculária coletada na Água Costeira (AC) e na Água Tropical (AT), na região de plataforma durante os dois períodos de coleta na área de estudo, bacia de Campos, RJ, Brasil.

\begin{tabular}{lcccccccccccc}
\hline & \multicolumn{4}{c}{ Chuvoso } & \multicolumn{3}{c}{ Seco } \\
\cline { 2 - 12 } & \multicolumn{3}{c}{ AC } & \multicolumn{3}{c}{ AT } & \multicolumn{3}{c}{ AC } & \multicolumn{3}{c}{ AT } \\
\cline { 2 - 11 } & Média & D.P. & $\%$ & Média & D.P. & $\%$ & Média & D.P. & $\%$ & Média & D.P. & $\%$ \\
\hline Oikopleura longicauda & 155,05 & 187,73 & 96,86 & 17,36 & 16,29 & 87,56 & 137,34 & 358,29 & 96,74 & 39,88 & 41,99 & 97,41 \\
Oikopleura rufescens & 0,19 & 0,33 & 0,12 & 1,12 & 1,45 & 5,66 & 0,41 & 1,34 & 0,29 & 0,20 & 0,27 & 0,48 \\
Oikopleura fusiformis & 1,24 & 2,77 & 0,78 & 0,62 & 0,87 & 3,13 & 1,06 & 2,37 & 0,75 & 0,54 & 0,38 & 1,32 \\
Oikopleura intermedia & 0,00 & 0,00 & 0,00 & 0,01 & 0,03 & 0,03 & 1,63 & 4,31 & 1,15 & 0,09 & 0,20 & 0,22 \\
Oikopleura cophocerca & 0,00 & 0,00 & 0,00 & 0,00 & 0,00 & 0,00 & 0,02 & 0,07 & 0,01 & 0,00 & 0,00 & 0,00 \\
Oikopleura cornutogastra & 0,00 & 0,00 & 0,00 & 0,00 & 0,00 & 0,00 & 0,13 & 0,52 & 0,09 & 0,00 & 0,00 & 0,00 \\
Oikopleura dioica & 0,00 & 0,00 & 0,00 & 0,00 & 0,00 & 0,00 & 0,24 & 0,92 & 0,17 & 0,00 & 0,00 & 0,00 \\
Fritillaria formica & 0,11 & 0,29 & 0,07 & 0,65 & 1,52 & 3,28 & 0,14 & 0,30 & 0,10 & 0,18 & 0,39 & 0,43 \\
Fritillaria pellucida & 3,25 & 8,60 & 2,03 & 0,01 & 0,04 & 0,07 & 1,00 & 2,33 & 0,70 & 0,00 & 0,00 & 0,00 \\
Fritillaria haplostoma & 0,24 & 0,63 & 0,15 & 0,00 & 0,00 & 0,00 & 0,01 & 0,06 & 0,01 & 0,06 & 0,13 & 0,14 \\
Fritillaria sargassi & 0,00 & 0,00 & 0,00 & 0,06 & 0,23 & 0,28 & 0,00 & 0,00 & 0,00 & 0,00 & 0,00 & 0,00 \\
\hline
\end{tabular}


Tab. III. Lista de espécies indicadoras $(\mathrm{p}<0,05)$, região/massa de água indicado, IndVal (\%) e resultado de significância do Teste de Monte Carlo (p) (AC, água costeira).

\begin{tabular}{lccc}
\hline & região/massa de água & IndVal & $\mathrm{p}$ \\
\hline O. longicauda & $\mathrm{AC}$ & 76.8 & 0.0002 \\
F. pellucida & $\mathrm{AC}$ & 29.6 & 0.0086 \\
O. cophocerca & Talude & 19.4 & 0.0300 \\
O. dioica & $\mathrm{AC}$ & 12.0 & 0.0286 \\
\hline
\end{tabular}

Na região de plataforma $O$. cophocerca, $O$. cornutogastra e $O$. dioica foram exclusivamente encontradas na AC e F. sargassi foi exclusiva da AT. Em ambas as massas de água na plataforma $O$. longicauda foi a mais abundante e chegou a representar $97,4 \%$ das apendiculárias na AT durante o período seco. As maiores densidades médias de $O$. longicauda, O. fusiformis, O. cophocerca, O. cornutogastra, $O$. dioica e F. pellucida foram superiores na AC. Algumas espécies, como F. formica e F. sargassi apresentaram maior densidade média na AT. Oikopleura rufescens, O. intermedia e $F$. haplostoma demonstraram maior densidade média em massa de água diferente durante os dois períodos de coleta (Tab. II). As variações na composição da comunidade das apendiculárias foram significativamente diferentes entre as massas de água AC e AT da plataforma nos dois períodos (nível de significância $=0,09 \%$ ).

Entre as apendiculárias encontradas, somente $O$. longicauda foi considerada bioindicadora da $\mathrm{AC}(\mathrm{p}=0,00 \mathrm{e}$ INDVAL $=76,80 \%$; Tab. III). Fritillaria pellucida, O. dioica e O. cophocerca também revelaram significância $(\mathrm{P}<0,05)$ com uma determinada massa de água ou região, porém seus valores de IndVal foram abaixo de $70 \%$ o que não caracteriza essas espécies como biondicadoras (Tab. III).

\section{DISCUSSĀO}

Os mecanismos de fertilização da zona eufótica na região costeira ocorrem através da contribuição de águas continentais que formam a AC ou através da intrusão da ACAS que caracteriza o processo de ressurgência, principalmente no verão (LEHMANN \& MYRBERG, 2008). A presença e a duração da resurgência costeira esta associada à intensidade dos ventos nordeste e a influência de vórtices (CASTELÃo et al., 2004), por isso, condições meteo-oceanográficas durante o período estudado provavelmente contribuíram para ausência da ACAS na superfície da plataforma continental na área estudada.

A maior densidade das apendiculárias, encontrada, na plataforma em relação ao talude e na $\mathrm{AC}$ em relação a AT, provavelmente está associada à maior influência da drenagem continental que possibilita uma maior concentração de nutrientes na plataforma, principalmente sobre influência da AC (ResGalla JR., 2001). Essa maior concentração de nutrientes favorece o aumento da produtividade primária e, consequentemente, da densidade dos organismos do zooplâncton que dela se alimentam (Hoover et al., 2006). Alguns autores já destacaram maiores abundâncias das apendiculárias em águas de plataforma, quando comparadas a águas oceânicas (Alldredge, 1976; ESNAL, 1999).

Os picos de abundância das apendiculárias variaram nos dois períodos, no chuvoso os maiores valores foram encontrados na região de Cabo Frio sobre influência da AC. Sabe-se que essa região é uma das mais produtivas da costa brasileira, devido ao fenômeno de ressurgência da ACAS nessa localidade (VALENTIN, 1984; VALENTIN et al., 1987; SiLVA et al., 2006). Apesar de não ter sido detectado a presença da ACAS na área e nos períodos de estudo, os altos valores de densidade das apendiculárias provavelmente se devem ao reflexo de um período de pós-ressurgência. Segundo Gonzalez-Rodrigues et al. (1992), no período de pósressurgência ocorre aumento na pastagem dos organismos zooplanctônicos na região de Cabo Frio.

No período seco os picos na abundância das apendiculárias foi observado na região norte da área de estudo influenciada pela AC. Essa região sofre forte influência da foz do rio Paraíba do Sul, cuja vazão pode interferir na produtividade local através da quantidade de matéria em suspensão e do aporte de nutrientes (SALOMÃo et al., 2001). Além disso, a maior produtividade dessa região normalmente é maior no período seco, quando ocorre diminuição da quantidade de material particulado na água e por consequência maior penetração de luz aumentando a produtividade dos organismos fitoplanctônicos autotróficos (AlmEIDA et al., 2007). Essa maior produtividade notadamente contribuiu para o aumento da densidade das apendiculárias no atual estudo.

Foram observados valores bem superiores de densidade quando se compara $O$. longicauda com as demais, nas duas regiões durante a influência das duas massa de água na plataforma. A informação sobre as altas frequências e densidades de $O$. longicauda é bem conhecida em vários lugares do mundo (ESNAL, 1999; BoneCKER \& CARVAlHO, 2006; HidaKa, 2008; Touzri et al., 2012); além disso, essa espécie é a mais encontrada em regiões costeiras e oceânicas (TомiтA et al. 2003; HidAKA, 2008), sendo no atual estudo mais encontrada na região de plataforma, sob influência da AC.

Oikopleura fusiformis foi a segunda mais representativa. Na baía de Toyama, no Japão (ToмiтA et al., 2003) e no Golfo do México esta espécie também foi a segunda mais abundante, logo atrás de O. longicauda, como neste estudo. Oikopleura fusiformis já foi encontrada como uma das mais frequentes na costa do Brasil, tanto em regiões costeiras quanto em oceânicas (BONECKER \& CARVALHO, 2006); apesar de ter sido observada nas duas regiões deste estudo, a espécie demonstrou preferência pela água costeira na região de plataforma.

Oikopleura dioica ocorreu somente na região de plataforma sobre influência da AC; a forte relação dessa espécie com o ambiente costeiro e até estuarino já foi descrito por diversos autores no Brasil e no mundo (p. ex. EsNAL, 1999; TOMITA et al., 2003; SHIGANOVA, 2005; BONECKER \& Carvalho, 2006; Carvalho \& Bonecker, 2010; Touzri et al., 2012). A ocorrência exclusiva de $O$. dioica na AC, água caracterizada por ser rica em nutrientes, pode estar relacionada ao fato de que essa espécie tem uma necessidade 
maior de alimentos do que as outras apendiculárias (ТомітA et al., 2003).

A maioria das espécies foi mais abundante em águas costeiras da região de plataforma, porém $O$. cophocerca, $O$. albicans, $O$. rufescens e $F$. formica apresentaram suas maiores densidades pelo menos em algum período no talude. Oikopleura cophocerca e O. albicans já foram citadas como raras nas regiões costeiras de Cabo Frio até o Rio Grande do Sul (ForNERIs, 1965), mas como no presente estudo, já foram encontradas em águas de plataforma e oceânicas na costa do Japão (HidAKa, 2008). Os maiores valores de densidade de $O$. rufescens foram encontrados sobre a influência da AT no período chuvoso. A preferência de $O$. rufescens por uma determinada massa de água superficial ainda não foi descrita, mas sabe-se que essa espécie é frequentemente encontrada em regiões costeiras (CARVALHO \& BONECKER, 2010) e oceânicas (HIDAKA, 2008) de águas quentes da região tropical (ARAVENA \& Palma, 2002). Fritillaria formica apresentou preferência pela AT; esta espécie é considerada oceânica (ZAKARIA, 2006) e assim como no atual estudo, apresentou preferência pela AT em águas oceânicas no sul do Brasil (ForNeris, 1965).

As apendiculárias de águas de plataforma diferiram das encontradas em águas oceânicas, tanto em termos qualitativos como quantitativos. Apesar da elevada produtividade primária tornar o zooplâncton costeiro muito abundante, fatores ambientais (como a ampla variação diária da salinidade e fatores biológicos) podem restringir a variedade das espécies quando comparado ao das áreas oceânicas (BRANDINI et al., 1997). Portanto, as diferenças encontradas na abundância e na composição das espécies de apendiculárias, provavelmente foram influenciadas pelas diferentes massas de águas superficiais do ambiente estudado.

As maiores abundâncias de $O$. longicauda na $\mathrm{AC}$ e relação a AT provavelmente foram determinantes para que essa espécie tenha sido indicadora dessa massa de água. Apesar de não existir estudos que mostrem a função de bioindicadora de O. longicauda, ESNAL (1999) apontou a preferência dessa espécie por águas costeiras. Em um estudo realizado na Região Sudeste do Brasil, próximo à área de estudo, $O$. longicauda também foi associada a águas costeiras (ESKINAZI-SANT'ANNA \& BJÖRNBERG, 2006). O teste ISA também evidenciou relações significativas de $O$. dioica e F. pellucida com a massa de água $\mathrm{AC}$ e de $O$. cophocerca com a região do talude, por apresentar baixos valores de IndVal $(<70 \%)$ essas espécies não foram consideradas bioindicadoras. Segundo McGeoch et al. (2002) somente espécies que apresentam valores de IndVal acima de 70\% são características ou indicadoras de um habitat, menores que isso são consideradas detectoras. Estudos com séries temporais mais longas podem ajudar a identificar o papel de bioindicador dessas espécies.

Vários fatores podem influenciar a variação das densidades das apendiculárias, como predação (CAPITANIO \& EsNaL, 1997) e a disponibilidade de alimento (DAGG \& Brown, 2005). O presente estudo mostrou que diferenças espaciais e de massa de água interferem diretamente na densidade e na composição específica das apendiculárias. Além disso, contribuiu para o conhecimento sobre as espécies bioindicadoras dessa classe. Outros estudos com maiores séries temporais são recomendados para a confirmação desses resultados e ampliação do conhecimento sobre a Classe Appendicularia e outros grupos zooplanctônicos.

Agradecimentos. Os autores agradecem à equipe do Laboratório Integrado de Zooplâncton e Ictioplâncton da Universidade Federal do Rio de Janeiro, a Dra Ana Bonecker pela revisão do trabalho e a Petrobras, que tornou possível a amostragem e análise do material.

\section{REFERÊNCIAS BIBLIOGRÁFICAS}

Alldredge, A. L. 1976. Discarded appendicularian houses as sources of food, surface habitats, and particulate organic matter in planktonic environments. Limnology and Oceanography 21:14-23.

Almeida, M. G.; Rezende, C. E. \& SouzA, C. M. M. 2007. Variação temporal, transporte e partição de $\mathrm{Hg}$ e carbono orgânico nas frações particulada e dissolvida da coluna d'água da bacia inferior do Rio Paraíba do Sul, RJ, Brasil. Geochimica Brasiliensis 21(1):111-128.

Aravena, G. \& Palma, S. 2002. Identificación taxonómica de las apendicularias capturadas en águas epipelágicas de la zona norte de Chile (Tunicata, Appendicularia). Revista Chilena de Historia Natural 75(2):307-325.

BJörnberG, T. K. S. \& Forneris, L. 1956. On the uneven distribution of the Copelata of the Alcatrazes area. Boletim do Instituto Oceanográfico 7(1-2):113-115.

Boltovskoy, D.; Correa, N. \& Boltovskoy, A. 2003. Marine zooplanktonic diversity: a view from the South Atlantic. Oceanologica Acta 25:271278.

Bonecker, S. L. C. \& Carvalho, P. F. 2006. Appendicularia. In: Bonecker, S. L. C. ed. Atlas da Região Central da Zona Econômica Exclusiva Brasileira. Rio de Janeiro, Museu Nacioinal, p.185-203.

Bonecker, S. L. C.; Araujo, A. V. De; Carvalho, P. F. De; Dias, C. De O.; Fernandes, L. F. L.; Migotto, A. E. \& Oliveira, O. M. P. De. 2014. Horizontal and vertical distribution of mesozooplankton species richness and composition down to 2,300 $\mathrm{m}$ in the southwest Atlantic Ocean. Zoologia 31(5):445-462.

Brandini, F. P.; Lopes, R. M.; Gutseit, K. S.; Spach, H. L. \& Sassi. R. 1997. Planctolologia na plataforma continental do Brasil. Diagnose e revisão bibliográfica. Rio de Janeiro, MMA-CIRM-FEMAR. 196p.

Capitanio, F. L. \& Esnal. G. B. 1997. Appendicularian distribution in the Rio de la Plata estuary and adjacent areas. Neritica Curitiva 11:37-48.

Capitanio, F.; PÁJaro, M. \& Esnal, G. B. 2005. Appendicularians: an important food supply for the Argentine anchovy Engraulis anchoita in coastal waters. Journal of Applied Ichthyology 21:414-419.

Carvalho, P. F. \& Bonecker, S. L. C. 2010. Seasonal and spatial variability of appendicularian density and taxonomic composition in the Caravelas Estuary (Northeastern Brazil) and adjacent coastal area. Brazilian Archives of Biology and Technology 53(1)161-169.

Castelão, R. M.; Campos, E. J. D. \& Miller, J. L. 2004. A modelling study of coastal upwelling driven by wind and meanders of Brazil Current. Journal of Coastal Research 20(3):662-671.

DAGG, M. J. \& Brown, S. L. 2005. The potential contribution of fecal pellets from larvaceans Oikopleura dioica to vertical flux of carbon in a river dominated coastal margin. In: GorsKy, G.; Youngbluth, M. J. \& DeIBEL, D. eds. Response of marine ecosystems to global change. Paris, Contemporary Publishing International, p.293-307.

Dias, C. O.; Araujo, A. V.; Vianna, S. C.; Loureiro Fernandes, F.; Paranhos, R.; Suzuki, M. S. \& Bonecker, S. L. C. 2015. Spatial and temporal changes in biomass, production and assemblage structure of mesozooplanktonic copepods in the tropical south-west Atlantic Ocean. Journal of the Marine Biological Association of the United Kingdom 95(3):483-496.

Dufrêne, M. \& LeGENDre, P. 1997. Species assemblages and indicator species: the need for flexible asymmetrical approach. Ecological Monographs 67(3):345-366. 
Eskinazi-SAnt'Anna, E. M. \& BuöRnBerg, T. K. S. 2006. Seasonal dynamics of mesozooplankton in Brazilian coastal waters. Hydrobiologia 563:253-268.

Esnal, G. B. 1999. Appendicularia. In: Boltovskoy, D. ed. South Atlantic Zooplankton. The Netherlands, Backhuys Publishers, p. 1375-1398.

EsPINO, G. L. 2000. Critérios generales para la elección de bioindicadores. In: Espino, G. L.; Pulido, S. H. \& PÉrez, J. L. C. eds. Organismos indicadores de la calidad del água y de la contaminación (bioindicadores). México, Plaza y Váldez, S.A. de C. V., p.17-41.

ForNeris, L. 1965. Appendicularian species groups and Southern Brazil water masses. Boletim do Instituto Oceanográfico 14:53-114.

Gonzalez-Rodriguez, E.; Valentin, J. L.; André, D. L. \& Jacob, S. A. 1992. Upwelling and downwelling at Cabo Frio (Brazil): Comparison of biomass and primary production responses. Journal of Plankton Research 14:289-306.

HIDAKA, K. 2008. Species composition and horizontal distribution of the appendicularian community in waters adjacent to the Kuroshio in winter-early spring. Plankton and Benthos Research 3:152-164.

Hoover, R. S.; Hoover, D.; Miller, M.; LANDry, M. R.; Decarlo, E. H. \& MACKENZIE, F. T. 2006. Zooplankton response to storm runoff in a tropical estuary: bottom-up and top-down controls. Marine Ecology Progress Series 318:187-201.

HsiaO, S. H.; KÂ, S.; FAnG, T. H. \& Hwang, J. S. 2011. Zooplankton assemblages as indicators of seasonal changes in water masses in the boundary waters between the East China Sea and the Taiwan Strait. Hydrobiologia 666:317-330.

Lehmann, A. \& Myrberg, K. 2008. Upwelling in the Baltic Sea - A review. Journal of Marine Systems 74:3-12

McGeoch, M. A.; Rensburg, B. J. V. \& Botes, A. 2002. The verification and application of biondicators: a case study of dung beetles in a savanna ecosystem. Journal of Applied Ecology 39:661-672.

Mémery, L.; Arhan, M.; Alvarez-Salgado, X. A.; Messia, M. J.; Mercier H.; Castro C. G.; \& Rios, A. F. 2000. The water masses along the western boundary of the south and equatorial Atlantic. Progress in Oceanography 47:69-98.

ResGalla JR., C. 2001. Estudo de impacto ambiental sobre a comunidade do zooplâncton na enseada do saco do Limões, baía sul da ilha de Santa Catarina, Brasil. Atlântica 23:5-16.
Salomão, M. S. M. B.; Molisani, M. M.; Ovalle, A. R. C.; Rezende, C. E.; Lacerda, L. D. E. \& Carvalho, C. E. V. 2001. Particulate heavy metal transport in the lower Paraíba do Sul River basin, southeastern, Brazil. Hydrological Processes 15:587-593.

Shiganova, T. 2005. Changes in appendicularian Oikopleura dioica abundance caused by invasion of alien ctenophores in the Black Sea. Journal of the Marine Biological Association of the United Kingdom 85:477-494.

Silva, G. L.; Dourado, M. S. \& CANDElla, R. N. 2006. Estudo preliminar da climatologia da ressurgência na região de Arraial do Cabo, RJ. Anais do XIV Congresso Brasileiro de Meteorologia. Florianópolis, Universidade Federal de Santa Catarina, p.1-11.

Silveira, I. C. A.; Miranda, L. B. DE \& Brown, W. S. 1994. On the origins of the North Brazi1 Current. Geophysical Research 99(C11):2250122512.

Silveira, I. C. A.; Schmidt, A. C. K.; Campos, E. J. D.; Godoi, S. S. \& IKEDA Y. 2000. A Corrente do Brasil ao largo da Costa Leste Brasileira. Revista Brasileira de Oceanografia 48(2):171-183.

Tomita, M.; ShigA, N. \& IkedA, T. 2003. Seasonal occurrence and vertical distribution of appendicularians in Toyama Bay, southern Japan Sea. Journal of Plankton Research 25(6):579-58

Touzri, C.; Hamdi, H.; Goy, J. \& Daly Yahia, M. N. 2012. Diversity and distribution of gelatinous zooplankton in the Southwestern Mediterranean Sea. Marine Ecology 33:393-406.

VALENTIN, J. L. 1984. Analyse des paramètres hydrobiologiques dans la remontée de Cabo Frio (Brésil). Marine Biology 82:259-276.

Valentin, J. L.; Monteiro-Ribas, M. A. \& Mureb, E. 1987. Sur quelques zooplanctontes abondants dans l'upwelling de Cabo Frio (Brésil). Journal of Plankton Research 9:1195-1226.

Vega-Pérez, L. M.; Campos, M. A. G. \& Schinke, K. P. 2011. Checklist of class appendicularia (Chordata: Tunicata) from São Paulo State, Brazil. Biota Neotropica 11(1a).

Viana, A. R.; Faugères, J. C.; Kowsmann, R. O.; Lima, J. A. M.; Caddah, L. F. G. \& Rizzo, J. G. 1998. Hydrology, morphology and sedimentology of the Campos continental margin, offshore Brazil. Sedimentary Geology 115:133-157.

ZAKARIA, H. Y. 2006. The zooplankton community in Egyptian Mediterranean Waters: A review. Acta Adriatica 47:195-206. 